\section{Dermoscopy of keratosis follicularis squamosa}

Michiyo Nakano, Naotomo Kambe, Takashi Satoh, Yaei Togawa,

Noriaki Kamada, Hiroyuki Matsue

Department of Dermatology, Chiba University Graduate School of Medicine, Chiba, Japan

\begin{abstract}
Although the characteristic clinical appearance and the differences in distribution, it is often difficult to differentiate keratosis follicularis squamosa (Dohi) from other keratotic disorders. Here, we describe the case of a 5 -year-old boy with Dohi in the first time that by using dermoscopy. Dermoscpoy of the lesion showed typical lotus leaves on the water appearance and follicular plug in the center. We reported the usefulness of dermosocopy in Dohi.
\end{abstract}

\section{Introduction}

Although the characteristic clinical appearance and the differences in distribution, it is often difficult to differentiate keratosis follicularis squamosa (Dohi) from other keratotic disorders. Here we reported in the first time that by using dermoscopy, atypical clinical appearance in Dohi became more obvious.

\section{Case Report}

A 5-year-old Japanese boy presented with a 1-year history of asymptomatic scaly eruptions symmetrically scattered on the trunk and thighs (Figure 1A). His medical history was unremarkable and he had no history of atopic dermatitis. There was no family history of a similar disease. Physical examination revealed small scattered patches whose margin is slightly detached from the underlying epidermis, but a typical brown-coloured follicular plug in the centre was not obvious (Figure 1B). However, dermoscopic observation without echo-jelly remarkably showed typical lotus leaves on the water appearance (Figure 2A). Moreover with echo-jelly, even though lotus leaves on the water appearance cannot observe, a typical brown-coloured follicular plug in the center and fatty hair which has larger diameter than normal area became obvious by dermascopy (Figure 2B). Histopatholo-gy showed dilated follicles with keratotic plugs and orthohyperkeratosis (Figure 2C). Treatment with 20\% uric acid containing cream led to clinical disappearance of the lamellar scaling improved.

\section{Discussion}

Dohi, characterized by $10 \pm 3 \mathrm{~mm}$ diameter scaly patches scattered on the trunk, is a keratinizing disorder first described by Dohi and Momose in $1903 .{ }^{1}$ Its asymptomatic scaly patches with a margin that is slightly raised from the underlying epidermis was originally described as lotus leaves on the water. A tiny pigmented papule is present in the center of the lesion. This is histopathologically a dilated hair follicle with a follicular plug surrounded by lamellar orthohyperkeratosis. Dohi is, thus, well recognized in Japan as a separate clinical entity from keratosis follicularis (Darier's disease). ${ }^{2}$ But it often diffi-

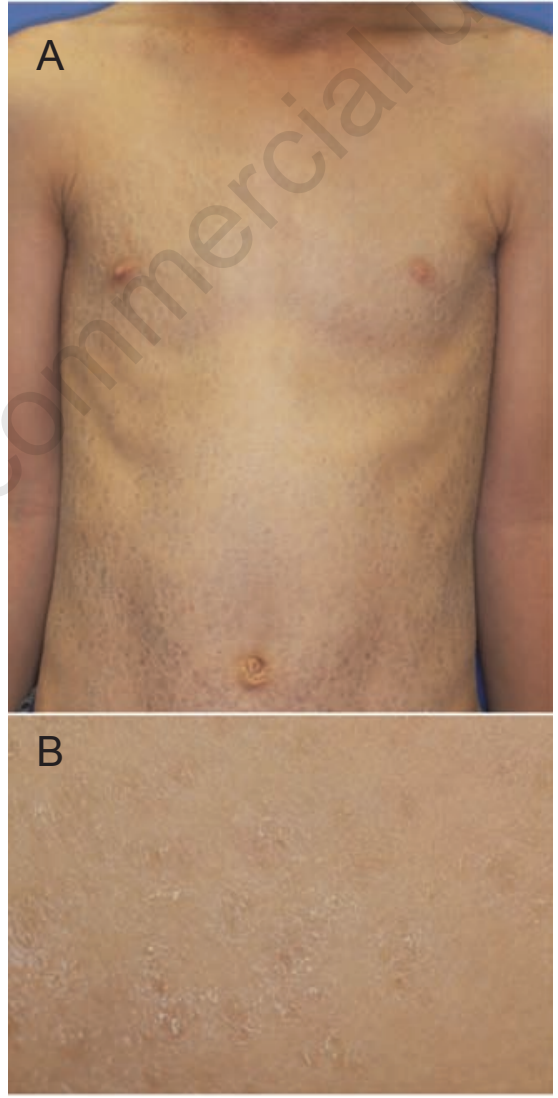

Figure 1. Clinical features of a patient with keratosis follicularis squamosa. A) Brown follicular papules surrounded by thin lamellar scales on the trunk. B) Typical lotus leaves on the water appearance are seen, but a typical brown-coloured follicular plug in the centre was not obvious.
Correspondence: Yaei Togawa, Department of Dermatology, Chiba University Graduate School of Medicine, 1-8-1 Inohana, Chuo-ku, Chiba 2608670 , Japan.

Tel. +81.43.226.2505 - Fax: +81.43.226.2128

E-mail: togawa-yk@faculty.chiba-u.jp

Key words: dermascopy, keratosis follicularis squamosal, Dohi.

Received for publication: 31 August 2011.

Accepted for publication: 31 August 2011.

This work is licensed under a Creative Commons Attribution NonCommercial 3.0 License (CC BYNC 3.0).

(OCopyright M. Nakano et al., 2011

Licensee PAGEPress, Italy

Dermatology Reports 2011; 3:e26

doi:10.4081/dr.2011.e26
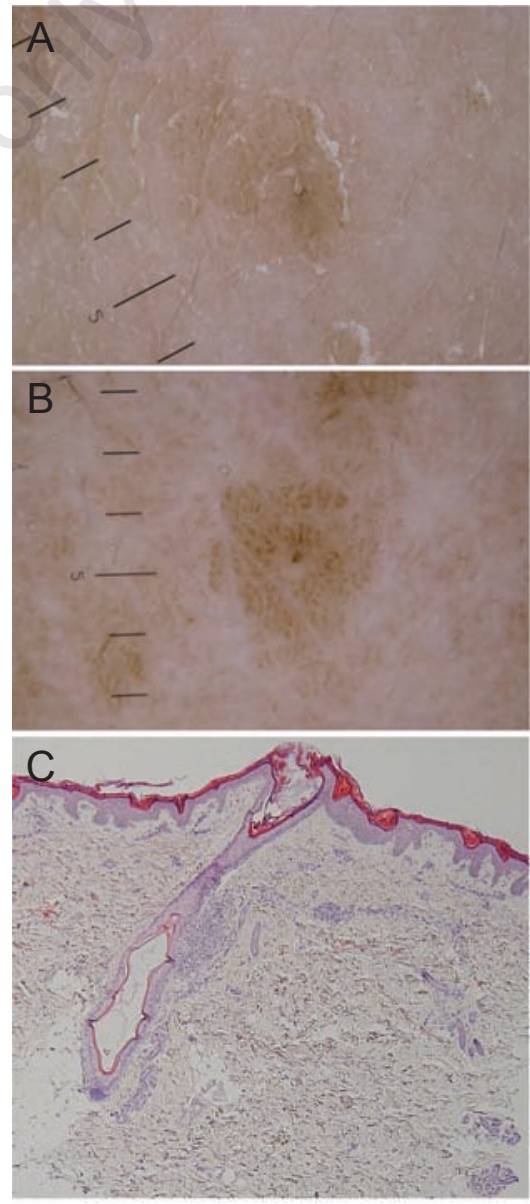

Figure 2. Dermoscopy observation of the lesion. A) Observation without echo-jelly remarkably represent typical lotus leaves on the water appearance. B) Observation with echo-jelly showed a typical browncoloured follicular plug in the center and delicate pigment network around it. C) Pathological findings of the lesion. A dilated hair follicle with keratotic plugging and surrounding epidermis with orthohyperkeratosis (haematoxylin and eosin, original magnification $\mathbf{x} 100$ ). 
cult to differentiate keratosis follicularis squamosa (Dohi) from other keratotic disorders when the characteristic feature of cutaneous findings are not obvious. Here we reported the usefulness of dermosocopy in Dohi.

\section{References}

1. Dohi K, Momose G. A presentation of patients with a certain follicular squamous keratosis (in Japanese). Jpn J Dermatol 1903;3:513-4.
2. Shimizu S, Shimizu T, Tateishi Y, Shimizu H. Keratosis follicularis squamosa (Dohi): a follicular keratotic disorder well known in Japan. Br J Dermatol 2001;144:1070-2. 